\title{
Efficacy of whole lung lavage in pulmonary alveolar proteinosis: a 20-year experience at a reference center in Thailand
}

\author{
Punchalee Kaenmuang, Asma Navasakulpong \\ Division of Respiratory and Respiratory Critical Care Medicine, Department of Internal Medicine, Faculty of Medicine, Prince of Songkhla \\ University, Hat Yai, Thailand \\ Contributions: (I) Conception and design: Both authors; (II) Administrative support: Both authors; (III) Provision of study materials or patients: P \\ Kaenmuang; (IV) Collection and assembly of data: P Kaenmuang; (V) Data analysis and interpretation: A Navasakulpong; (VI) Manuscript writing: \\ Both authors; (VII) Final approval of manuscript: Both authors. \\ Correspondence to: Assistant Professor Asma Navasakulpong. Division of Respiratory and Respiratory Critical Care Medicine, Department of Internal \\ Medicine, Faculty of Medicine, Prince of Songkhla University, Hat Yai, Songkhla 90110, Thailand. Email: navasakulpong@hotmail.com.
}

\begin{abstract}
Background: Pulmonary alveolar proteinosis (PAP) is a rare lung syndrome. The current standard treatment is whole lung lavage (WLL). We reviewed PAP cases treated with WLL during a 243-month period. The primary objective was to describe the efficacy of WLL. We compared chest imaging resolution and pulmonary function tests $\left(\mathrm{PaO}_{2}\right.$ and DLCO) before the first and after the last WLL. The secondary objectives were to compare mMRC dyspnea scores, other lung function parameters, and complications of WLL.

Methods: We retrospectively reviewed PAP patients from 1 January 2000 to 31 March 2020. Demographic data, pulmonary function tests, and the efficacy of WLL were collected from the electronic medical database and analyzed by descriptive analysis. Differences in data used the student $t$-test to compare parameters pre- and post-WLL.

Results: A total of 19 PAP patients and 50 WLL procedures were included. Eleven patients (57.9\%) were females and the mean age was $51.5 \pm 11.7$ years. Dyspnea $(100 \%)$ and cough $(94.7 \%)$ were the two leading symptoms. The most common indication for WLL was progressive dyspnea. There were significant improvements in $\mathrm{SpO}_{2}$ from $86 \%$ to $94 \%$ ( $\left.\mathrm{P}<0.001\right), \mathrm{PaO}_{2}$ from 49.3 to 66.1 ( $\left.\mathrm{P}<0.001\right)$, DLCO from $31.8 \%$ to $52.5 \%$ predicted $(\mathrm{P}=0.013)$, and the $\mathrm{mMRC}$ dyspnea score from 3 to $2(\mathrm{P}<0.001)$ without major complications.
\end{abstract}

Conclusions: WLL is an effective standard treatment for PAP cases. It is safe and can be used as a primary treatment in case of inhaled anti GM-CSF is not available.

Keywords: Pulmonary alveolar proteinosis (PAP); whole lung lavage (WLL); surfactant

Submitted Nov 14, 2020. Accepted for publication May 14, 2021.

doi: 10.21037/jtd-20-3308

View this article at: http://dx.doi.org/10.21037/jtd-20-3308

\section{Introduction}

Pulmonary alveolar proteinosis (PAP) was first described in 1958 by Rosen (1). It is a syndrome characterized by the accumulation of alveolar surfactant and dysfunction of alveolar macrophages. PAP can be classified into one of three categories: primary, secondary, and congenital PAP. Primary PAP is characterized by the disruption of granulocyte-macrophage colony-stimulating factor (GMCSF) signalling and can be autoimmune caused by elevated levels of GM-CSF autoantibodies, which accounts for $>90 \%$ of cases, or hereditary (due to mutations in CSF $2 R A$ or CSF2RB, encoding GM-CSF receptor subunits). Secondary PAP results from various underlying conditions. The third category is congenital PAP which is caused by mutations in genes involved in surfactant production (2-4). 


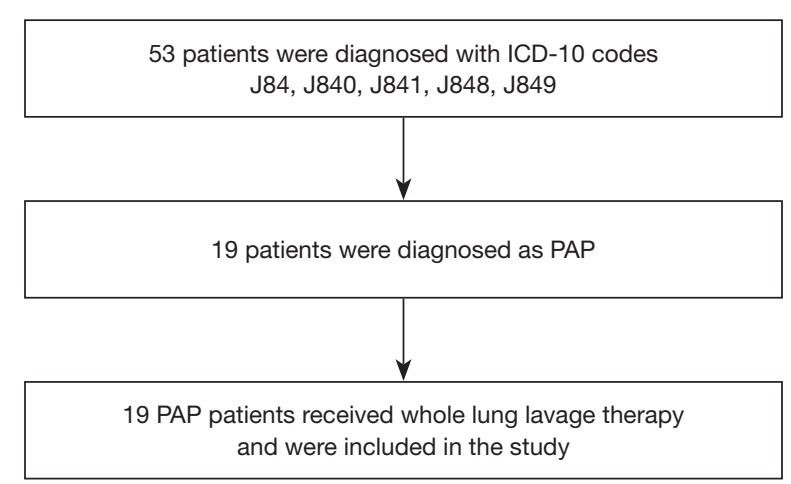

Figure 1 Patient flow diagram. PAP, pulmonary alveolar proteinosis.

For patients with moderate to severe symptoms, whole lung lavage (WLL) is still the first-line treatment (5). Although supplemental GM-CSF is also very useful for autoimmune PAP, it is not available worldwide and the new promising treatments, such as anti-CD20 monoclonal antibody, therapeutic plasma exchange, and lung transplant, are reserved for refractory cases (6-8). Since the experience of managing such patients is limited only to case reports and case series studies, limited data are available in Thailand, Asia, and worldwide. We have managed several PAP patients who were either new or referred patients. The aim of our study was to evaluate the efficacy of WLL and extensively review the characteristics, clinical presentations, radiological findings, and prognosis of these patients.

We present the following article in accordance with the STROBE reporting checklist (available at http://dx.doi. org/10.21037/jtd-20-3308).

\section{Methods}

\section{Study design and patients}

This retrospective study extracted PAP patients using ICD10 codes J84, J840, J841, J848, and J849 from a health information database system at Songklanagarind Hospital from 1 January 2000 to 31 March 2020. All cases were reviewed by the authors. Eligible cases included patients who were (I) $>18$ years old, (II) diagnosed as PAP by highresolution computed tomography (HRCT)/CT chest and bronchoalveolar lavage (BAL) with or without tissue biopsy, and (III) treated with WLL (Figure 1). Patients who did not undergo WLL were excluded from this study. Our study was conducted in accordance with the Declaration of Helsinki (as revised in 2013), approved by the Office of Human Research Ethics Committee, Faculty of Medicine,
Prince of Songkhla University (REC 61-194-14-1) and individual consent for this retrospective analysis was waived.

We conducted this study to collect all baseline characteristics of the PAP patients and data of WLL procedures. The primary objective was to describe the efficacy of WLL in PAP patients by comparing the chest radiographs or CT chest and pulmonary function tests, including arterial oxygen partial pressure $\left(\mathrm{PaO}_{2}\right)$ and diffusing capacity for carbon monoxide (DLCO), between before the first and after the last WLL. The secondary objective was to compare the modified Medical Research Council (mMRC) dyspnea scores and other lung function parameters $\left[\mathrm{FEV}_{1} / \mathrm{FVC}\right.$ ratio, FVC, total lung capacity, and the DLCO/alveolar volume (VA) ratio] between before the first and after the last WLL, and complications of WLL. We followed up all patients in the medical records to eliminate potential sources of bias.

\section{Disease severity score (DSS) and stratification of the patients}

Patients were assigned a PAP DSS based on the presence of symptoms (dyspnea or cough) and a reduction of $\mathrm{PaO}_{2}$ in room air, at rest, and supine position at the initial presentation as previously described (9). The categories were: DSS $1=$ no symptoms and $\mathrm{PaO}_{2} \geq 70 \mathrm{mmHg}$; DSS $2=$ symptomatic and $\mathrm{PaO}_{2} \geq 70 \mathrm{mmHg}$; DSS $3=70 \mathrm{mmHg}$ $<\mathrm{PaO}_{2} \geq 60 \mathrm{mmHg}$; DSS $4=60 \mathrm{mmHg}>\mathrm{PaO}_{2} \geq 50 \mathrm{mmHg}$; DSS $5=\mathrm{PaO}_{2}<50 \mathrm{mmHg}$ (Table 1).

\section{Whole lung lavage procedure (10)}

The WLL technique is performed under general anaesthesia in an operating room. The patient is intubated with a 
Table 1 Demographic and clinical characteristics of PAP patients

\begin{tabular}{|c|c|}
\hline Parameters & Patients $(n=19)$ \\
\hline Female & $11(57.9)$ \\
\hline Age of onset (years), mean \pm SD & $51.5 \pm 11.7$ \\
\hline Body mass index $\left(\mathrm{kg} / \mathrm{m}^{2}\right)$, median $\left(Q_{1}, Q_{3}\right)$ & $22.7(19.8,24.3)$ \\
\hline \multicolumn{2}{|l|}{ Smoking status } \\
\hline Current smokers & $1(5.3)$ \\
\hline Former smokers & $7(36.8)$ \\
\hline Non-smokers & $11(57.9)$ \\
\hline \multicolumn{2}{|l|}{ Clinical symptoms (mutually exclusive) } \\
\hline Cough & $18(94.7)$ \\
\hline Dyspnea on exertion & $19(100)$ \\
\hline Weight loss & $13(68.4)$ \\
\hline Fatigue & $13(68.4)$ \\
\hline Fever & $2(10.5)$ \\
\hline \multicolumn{2}{|l|}{ Physical examination (mutually exclusive) } \\
\hline Digital clubbing & $15(78.9)$ \\
\hline Crackles & $11(57.9)$ \\
\hline Unremarkable & $4(21.1)$ \\
\hline Time to diagnosis (months), median $\left(\mathrm{Q}_{1}, \mathrm{Q}_{3}\right)$ & $4(1.3,5.8)$ \\
\hline \multicolumn{2}{|l|}{ Diagnostic method } \\
\hline $\begin{array}{l}\text { Transbronchial biopsy and bronchoalveolar } \\
\text { lavage }\end{array}$ & $19(100.0)$ \\
\hline \multicolumn{2}{|l|}{ Etiologies } \\
\hline Primary PAP & $13(68.4)$ \\
\hline \multicolumn{2}{|l|}{ Secondary PAP } \\
\hline Active TB infection & $1(5.3)$ \\
\hline Previous TB infection & $4(21.1)$ \\
\hline Bacterial infection & $1(5.3)$ \\
\hline \multicolumn{2}{|l|}{ Disease involvement } \\
\hline Bilateral & $18(94.7)$ \\
\hline Unilateral & $1(5.3)$ \\
\hline \multicolumn{2}{|l|}{ DSS } \\
\hline DSS 1 & 0 \\
\hline DSS 2 & $1(5.0)$ \\
\hline DSS 3 & $7(37.0)$ \\
\hline DSS 4 & $6(32.0)$ \\
\hline DSS 5 & $5(26.0)$ \\
\hline Pulmonary arterial hypertension & $2(11.1)$ \\
\hline
\end{tabular}

Data are presented as $\mathrm{n}(\%)$ unless indicated otherwise. PAP, pulmonary alveolar proteinosis; SD, standard deviation; Q1, Q3, interquartile range; TB, tuberculosis; DSS, disease severity score. double-lumen endotracheal tube (DL-ETT) and fiberoptic bronchoscopy is performed to confirm appropriate tube placement. The patient is placed in the lateral decubitus position. The lung is then lavaged in the uppermost position while the non-lavaged lung is mechanically ventilated. Warmed saline $\left(37^{\circ} \mathrm{C}, 500-1,000 \mathrm{~mL}\right)$ is injected into the lung per one cycle. Fluid is then collected by gravity after opening the outflow tube. Manual chest percussion may be performed to improve drainage. Lavage and percussion are continued until the outflow fluid becomes definitively clear (Figure 2), which may take several hours and a total of 15-20 L of saline for a single lung lavage. After completion of WLL, two-lung ventilation is resumed and the DLETT is exchanged for a single-lumen endotracheal tube. The patient is then sent directly to the recovery room or respiratory care unit. If the hemodynamics and gas exchanges allow, some patients can be extubated on the same day.

\section{Statistical analysis}

Descriptive data are presented as mean \pm standard deviation (SD), median [interquartile range $\left(\mathrm{Q}_{1}, \mathrm{Q}_{3}\right)$ ] and number with percent (\%) based on the type of data. All continuous variables are presented as mean $\pm \mathrm{SD}$ or median $\left(\mathrm{Q}_{1}, \mathrm{Q}_{3}\right)$. Categorical variables are presented as either percentage of the total or numerically, as appropriate. For comparisons we used the student $t$-test for continuous variables. The statistical analysis used R 3.5.2 software and the results were considered statistically significant if the $\mathrm{P}$ value was $<0.05$.

\section{Results}

During the 243-month study period, a total of 19 PAP patients were included into the study. Demographic and clinical characteristics at baseline are presented in Table 1. Eleven $(57.9 \%)$ patients were females and the mean \pm SD age was $51.5 \pm 11.7$ years old. Most patients were nonsmokers $(57.9 \%)$. A smoking history was found in eight patients $(42.1 \%)$. The median time to diagnosis was four months. Dyspnea (100\%) and a cough $(94.7 \%)$ were the two leading clinical symptoms. Digital clubbing was usually found on physical examination (78.9\%), whereas four patients had unremarkable breath sounds and absence of digital clubbing. All patients were diagnosed as PAP according to the clinical, radiological, BAL, and histopathologic findings. Transbronchial biopsy and BAL were available in all cases for the definite diagnosis and 

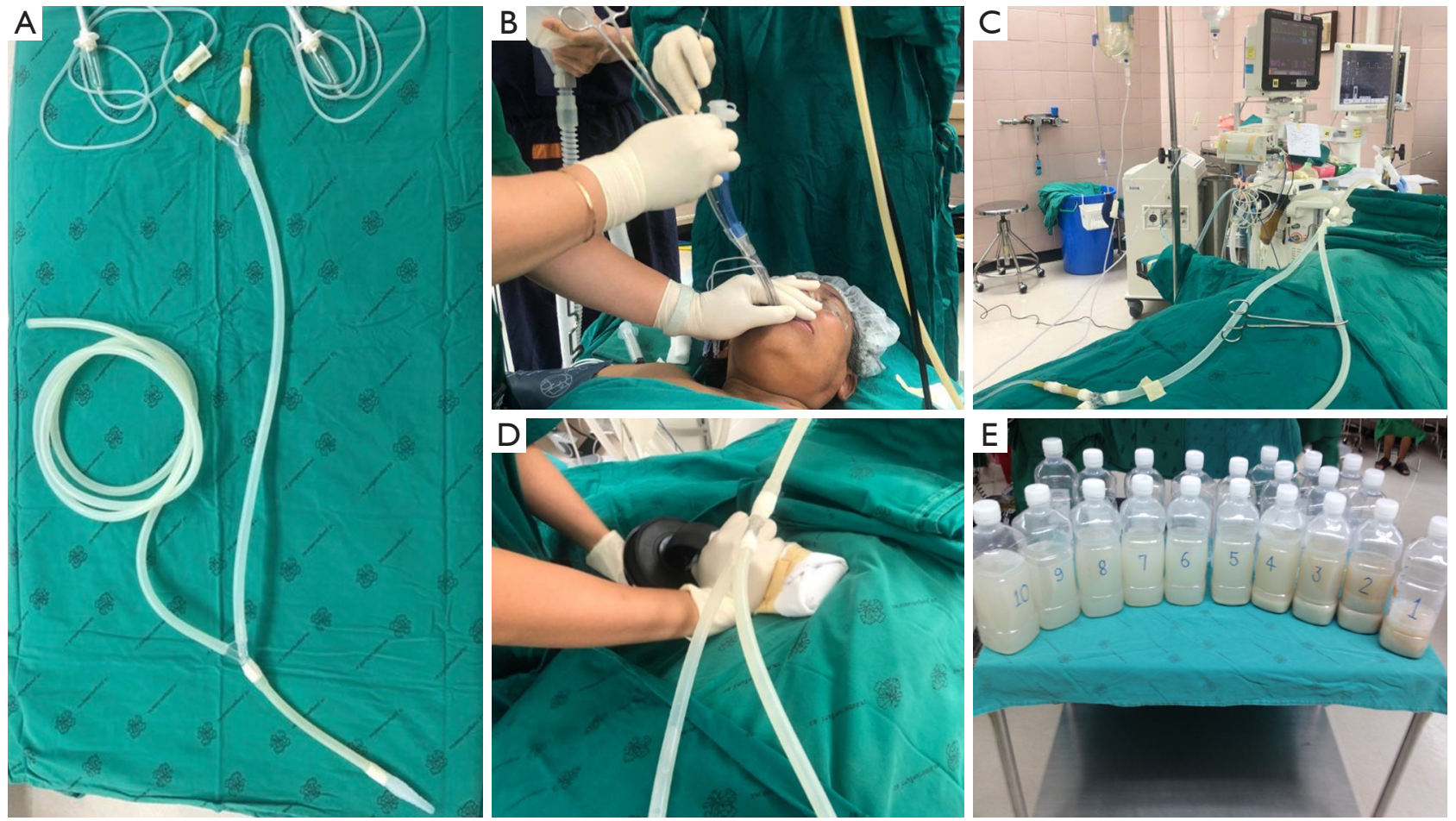

Figure 2 Whole lung lavage procedure (A) Lavage equipment consisted of inflow limb, outflow limb, and a portion to connect with the double-lumen endotracheal tube (B) The patient is intubated with a double-lumen endotracheal tube and fiberoptic bronchoscopy is performed to confirm the appropriate tube placement. (C) Warmed saline $\left(37^{\circ} \mathrm{C}, 500-1,000 \mathrm{~mL}\right)$ is injected in the lung. Fluid is then collected by gravity after opening the outflow tube. (D) Manual chest percussion may be performed to improve drainage. (E) Milky fluid extracted from the lung of a PAP patient in each cycle of the WLL procedure until the outflow fluid became definitively clear in the last cycle.

to identify secondary causes such as bacterial infection, tuberculosis or solid/hematologic malignancy. Six (31.6\%) patients had secondary PAP, mostly from previous tuberculosis infection. Thirteen PAP patients were suspected primary PAP; however, testing for anti-GMCSF antibodies, serum GM-CSF level, and GM-CSF signalling are not available in Thailand. Most of the PAP patients had bilateral lung involvement and two patients had secondary pulmonary hypertension defined as a mean pulmonary arterial pressure $>25 \mathrm{mmHg}$ from right heart catheterization.

A total of $50 \mathrm{WLL}$ procedures were performed (Table 2). Most of the patients were intubated with a left-sided double-lumen tube. The median number of WLL procedures per case was four and the median number of WLL procedures per case per year was two. Four cases needed only a single WLL. The maximum number of WLL procedures per case was 16 in 8 years. The median duration of the WLL procedure was $205.6 \pm 65.7$ minutes. The median (IQR) total lavage volume was 9,450 (7,352, $10,000) \mathrm{mL}$ and the median (IQR) net fluid balance was $-1,380(823,1,812) \mathrm{mL}$.

Diuretics were given according to the pulmonologists' opinion, mostly in case of positive fluid balance during the WLL procedures. Invasive mechanical ventilators were used post-WLL in 42 procedures (84\%), the median time for mechanical ventilator was five hours after the procedures. Eight patients ( $42 \%$ ) were extubated after the WLL procedure. Six patients used a face mask after extubation and two patients used a non-invasive mechanical ventilator. The procedure complications were refractory hypoxemia in $12(24 \%)$ procedures, pneumonia in $5(10 \%)$ procedures, and bronchospasm in $5(10 \%)$ procedures. Hypotension was found in $4(8 \%)$ procedures and these patients needed temporary vasopressors which were discontinued within 24 hours after WLL. The median length of hospital stay was 
Table 2 Whole lung lavage procedures

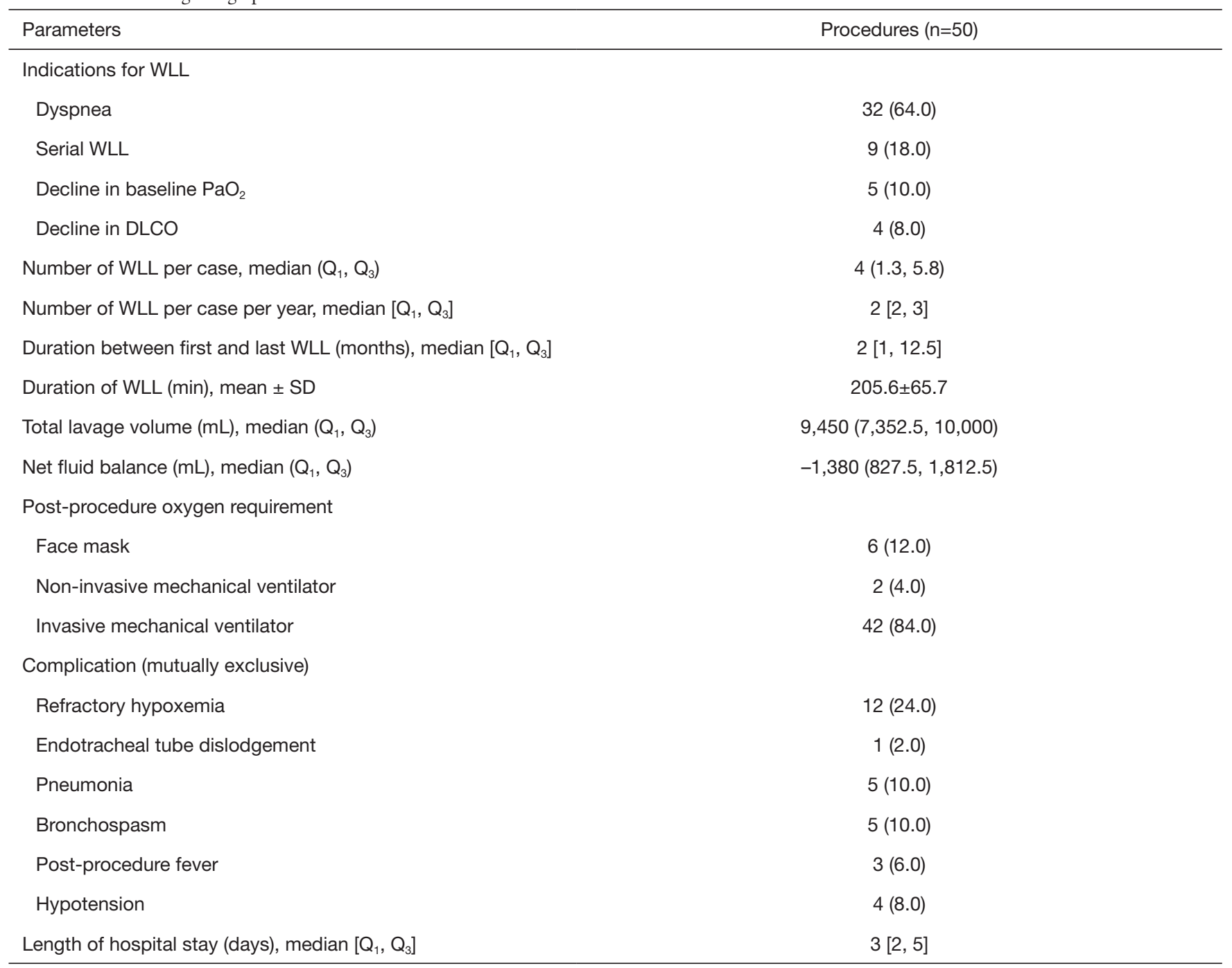

Data are presented as $\mathrm{n}(\%)$ unless indicated otherwise. WLL, whole lung lavage; $\mathrm{PaO}_{2}$, arterial oxygen partial pressure; DLCO, diffusing capacity for carbon monoxide; Q1, Q3, interquartile range; SD, standard deviation.

three days.

The lung parameters, oxygenation and DLCO before the first and after the last WLL, are shown in Table 3. There were significant improvements in oxygen saturation $\left(\mathrm{SpO}_{2}\right)$ from $86 \%$ to $94 \%(\mathrm{P}<0.001), \mathrm{PaO}_{2}$ from 49.3 to $66.1 \mathrm{mmHg}(\mathrm{P}<0.001)$, and in DLCO from $44.8 \%$ to $59.5 \%$ predicted $(\mathrm{P}=0.013)$. We also used the mMRC dyspnea score as a parameter. A significant improvement of the mMRC dyspnea score from 3 to 2 was observed in our study $(\mathrm{P}<0.001)$.

During follow-up, imaging resolution using either chest radiograph or HRCT of the chest and clinical outcomes of the PAP patients are shown in Table 4. Complete improvements were observed in the thoracic imaging after the last WLL in nine $(47.37 \%)$ patients and partial improvement in eight $(42.1 \%)$ patients. We followed up all patients who were still alive. The median time of followup was 5.8 years. A Kaplan-Meier curve was shown the probability of still being in follow-up. There were 19, 12, 4, and 1 patients at baseline, 5, 10, and 15 years, respectively (Figure 3). The longest follow-up was 18 years in one patient. Up to the present, the patient still had no abnormal symptoms since the last WLL which was done ten years previously. Five-year survival was $63 \%$ in 12 PAP patients who were followed for more than five years. Two patients had no improvement of imaging resolution and died due 
Table $3 \mathrm{mMRC}$ dyspnea score, oxygen saturation, and pulmonary function tests at pre- and post-lung lavage (last cycle)

\begin{tabular}{lccc}
\hline Parameters & Pre-WLL & Post-last WLL & P value \\
\hline mMRC dyspnea score, median $\left(\mathrm{Q}_{1}, \mathrm{Q}_{3}\right)$ & $3[3,4]$ & $2[1,2]$ & $<0.001$ \\
$\mathrm{SpO}_{2}(\%)$ & $86.2 \pm 8.8$ & $94.0 \pm 6.6$ & $<0.001$ \\
$\mathrm{PaO}_{2}(\mathrm{mmHg})$ & $49.3 \pm 21.6$ & $66.1 \pm 27.5$ & $<0.001$ \\
FEV1 $(\%$ predicted) & $51.6 \pm 30.8$ & $64.1 \pm 31.0$ & 0.301 \\
FEV1 (L) & $1.3 \pm 0.9$ & $1.5 \pm 0.9$ & 0.091 \\
FEV1/FVC ratio (\%) & $59.7 \pm 38.5$ & $76.8 \pm 10.5$ & 0.712 \\
FVC (\% predicted) & $69.2 \pm 14.0$ & $74.5 \pm 14.6$ & 0.111 \\
TLC (\% predicted) & $73.4 \pm 17.5$ & $75.0 \pm 14.6$ & 0.919 \\
DLCO (mL/mmHg/min) & $44.8 \pm 16.9$ & $59.5 \pm 9.7$ & 0.013 \\
\hline
\end{tabular}

Data are presented as mean \pm SD unless indicated otherwise. mMRC, modified Medical Research Council; Q1, Q3, interquartile range; $\mathrm{SpO}_{2}$, oxygen saturation; $\mathrm{PaO}_{2}$, arterial oxygen partial pressure; FEV1, forced expiratory volume in one second; FVC, forced vital capacity; TLC, total lung capacity; DLCO, diffusing capacity for carbon monoxide.

Table 4 Follow-up outcomes

\begin{tabular}{lc}
\hline Outcomes & Patients \\
\hline Imaging resolution & \\
Complete Improvement & $9(47.4)$ \\
Partial improvement & $8(42.1)$ \\
Unchanged & $2(10.5)$ \\
Follow-up duration, (years), median $\left(\mathrm{Q}_{1}, \mathrm{Q}_{3}\right)$ & $5.8(1.5,9.8)$ \\
Mortality & $2(11.1)$ \\
\hline
\end{tabular}

Data are presented as $n(\%)$ unless indicated otherwise.

to disease progression. The first patient was a 61-year-old female and received three WLL procedures. After nine years of follow-up, she developed recurrent dyspnea with right heart failure. Her HRCT showed chronic PAP with bronchiectatic and fibrotic changes. She was admitted due to severe pulmonary hypertension and cor pulmonale, but she refused to undergo further WLL. The second patient was a 69-year-old female with pulmonary hypertension and cor pulmonale. She received three WLL procedures every three months but her chest images did not show improvement. Her HRCT showed chronic PAP with fibrotic lung disease. After the fourth WLL, she developed severe persistent hypoxemia and hypotension which did not

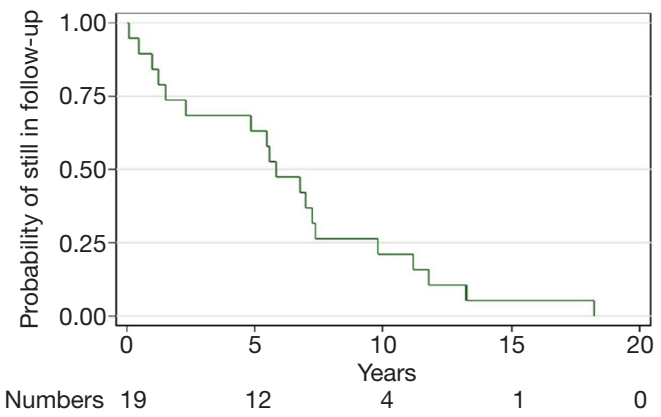

Figure 3 Kaplan-Meier Curve shows the probability of still being in follow-up. The longest follow-up period from diagnosis of PAP was investigated in each case. PAP, pulmonary alveolar proteinosis.

respond to treatment.

\section{Discussion}

This study is the first case series in Thailand describing the clinical characteristics of PAP patients, details of the WLL procedure, and the efficacy and complications of WLL in the context of an extremely rare set of symptoms in our country and in Southeast Asia. The efficacy, safety, and the lack of a standardized WLL procedure are infrequently reported in Southeast Asia. Our PAP patients had some differences in the baseline clinical characteristics compared to European, Japanese, Chinese, Italian, and Korean 
Table 5 Imaging resolution according to DSS

\begin{tabular}{lcccc}
\hline $\begin{array}{l}\text { Imaging } \\
\text { resolution }\end{array}$ & DSS 2 & DSS 3 & DSS 4 & DSS 5 \\
\hline No resolution & 0 & 0 & 2 & 0 \\
Partial & 0 & 5 & 3 & 1 \\
Complete & 1 & 2 & 1 & 4 \\
Total, n (\%) & $1(5.3)$ & $7(36.8)$ & $6(31.6)$ & $5(26.3)$ \\
\hline
\end{tabular}

DSS, disease severity score.

studies (9-16). Most of the PAP patients in our study were females which was different from previous studies $(9,10,13-16)$. The median age in our study was similar to Inoue et al. and Byun et al. $(9,12)$ but was different from Seymour et al. and $\mathrm{Xu}$ et al. who reported median ages at diagnosis of 39 years in the male patients and 35 years in the female patients and 42 years, respectively $(2,13)$. In our study, a "typical patient" was a male smoker aged 30 to 50 years and females aged 25 to 40 were underrepresented. The mean $\pm \mathrm{SD}$ ages of the males and females were $47.6 \pm 8.9$ and $54 \pm 12.6$ years old, respectively. Since most of our patients were female, that might explain the high percentage of non-smokers in our study which is different from previous studies (10). The percentages of PAP patients who were never-smokers ranged from $21 \%$ in a German series to $43 \%$ in a Japanese cohort. However, we did not address potential effects of passive smoke exposure in the female group. When we compared smokers and non-smokers, we found that the median (IQR) times of WLL per case were $2.5(0.5,3.5)$ times in smokers and $2(1.5,2.5)$ times in non-smokers which was similar to previous studies where smokers required repeat WLL more often than non-smokers $(16,17)$. Our patients were diagnosed rather early at 4 months after initial presentation. This was different from Seymour et al. and Inoue et al. who reported median durations of symptoms before diagnosis of 7 and 10 months, respectively $(2,9)$.

The interesting issue was that tuberculous infection, either active or previous infection, was the major cause of secondary PAP in our study which was not reported in other studies. The clinical presentations in our study (dyspnea on exertion and cough) were similar to the study by Inoue et al. (9). Their study reported 248 PAP patients in Japan who commonly presented with dyspnea and cough and one-third of the patients were asymptomatic. Also, Briens et al. (18) reported a GERMOP multi-centre retrospective study of 41 patients in France where dyspnea was the most common presenting symptom (54.3\%) while $31.8 \%$ of the patients were asymptomatic and were identified by health screening. However, only $5 \%$ of the patients were asymptomatic PAP in a study by Campo et al. (10). In our study, all patients were symptomatic and needed WLL treatment. A correlation existed between the DSS and DLCO. A more severe disease resulted in a more decreased DLCO. The resolution of imaging was different from a previous study (16). The complete resolution of imaging abnormalities in our study was found in up to $80 \%$ in DSS 5 group, while the previous study showed the resolution of imaging was found mostly in the DSS 3 group (Table 5). Possibly the degree of resolution of imaging is not correlated with the degree of disease severity at the beginning. We also found that the two cases that died had no improvement in the imaging and both had pulmonary arterial hypertension. We thought that the pulmonary arterial hypertension might be a poor prognostic factor for PAP patients.

We routinely perform WLL as the first-line treatment for symptomatic patients with moderate to severe disease (DSS 2-5). We had 50 procedures in 19 patients. The most common reason to perform WLL was dyspnea associated with respiratory insufficiency which is similar to a previous study (15). The most affected lung from chest imaging was lavaged first. Using this approach allows for single-lung ventilation of the less diseased lung initially and produces improved gas exchange in the more diseased lung following lavage. Nine (18\%) patients had serial WLL due to extensive bilateral lung infiltrations. None of those patients had both lungs treated in the same setting. We do a serial lavage to complete the initial therapeutic approach which was similar to other studies $(13,16,19)$. Only one procedure underwent WLL with a flexible bronchoscope because intubation was not possible with a double-lumen tube and the patient did not have a severe disease. The left-sided DLETT was preferred in our study due to concerns with rightsided DL-ETT migration producing spillage of lavage fluid and insufficient WLL of the right lung due to inadequate access to the right upper lobe. Four patients (44\%) needed only a single WLL to improve symptoms and sustain longterm benefit in respiratory function. The percentage of patients who needed a single WLL in our study was similar to several studies where $30 \%$ to $50 \%$ of patients required only one WLL $(16,19,20)$. However, our results were lower than the Campo et al. (10) study that reported $70 \%$ of the cases had only a single WLL during two decades of 
follow-up. Additionally, we had one patient who needed 16 WLL procedures in 8 years and was still alive. We believe that our primary PAP patients had autoimmune PAP, but unfortunately, we did not have the serum GM-CSF autoantibody test. The rate of primary PAP in our study was lower than in other studies that reported more than $90 \%$ of the cases were primary PAP and autoimmune PAP (3). The reasons to explain our results were (I) we are in an endemic area of tuberculosis that can cause secondary PAP and (II) occupational exposure to pesticides and herbicides may be related to PAP pathogenesis. The majority of our patients were farmers who cultivate rice and rubber. The chances of repeated exposure to organic dust, pesticides, and herbicides were quite high in these patients which might explain the reason the median number of WLL procedures per case in our study was higher than in previous a study (10). The mean \pm SD duration of WLL was $205.64 \pm 65.7$ minutes which was longer than previous studies $(11,15)$. The possible explanation is the use of mechanical chest percussion which was used to enhance the drainage of PAP sediment and fluid. The median (IQR) amount of lavage volume in our study was $9,450(7,352.5,10,000) \mathrm{mL} /$ time which was lower than the studies from Europe $(12,000 \mathrm{~mL})$ and America $(15,262 \pm 5,761 \mathrm{~mL})(11,15)$. This could be because the Asian population is smaller in size and stature. The median (IQR) net fluid balance was negative $[-1,380(827.5,1,812.5 \mathrm{~mL})]$ because $35(70 \%)$ of the WLL procedures were administered diuretics during the procedure. Forty-two (84\%) procedures needed a short interval of post-procedure invasive mechanical ventilation. All of these patients had bilateral lung infiltrations (DSS 4). In eight procedures, the patients (DSS 3) could be extubated immediately after the procedure. Upon finishing the procedure, all patients were transferred to the respiratory care unit. The median length of hospital stay during admission was 3 days which was quite similar to previous studies $(11,15)$.

Baseline pulmonary function tests in all patients indicated restrictive lung defect with diffusion defect, a mean predicted $\mathrm{FVC}$ of $69.2 \%$ predicted and $\mathrm{FEV}_{1}$ of $51.6 \%$ predicted. The mean diffusing capacity declined to $44.8 \%$ predicted. After the last WLL procedure, significant improvements were observed in oxygenation $\left(\mathrm{SpO}_{2}\right.$ and $\left.\mathrm{PaO}_{2}\right)$, DLCO, and in the mMRC dyspnea score in our patients. Other lung function parameters, such as the FVC, $\mathrm{FEV}_{1}$, and $\mathrm{FEV}_{1} / \mathrm{FVC}$, were not significantly different. The findings in our study were similar to many studies (12-15). We also compared chest imaging resolution and found that $17(89.5 \%)$ patients had improved chest imaging after the last WLL which was a higher rate compared with Smith et al. (62\%) (11). Two (10.5\%) patients, who had no improvement in the chest imaging, had chronic progressive disease, and their HRCT findings revealed fibrotic lung diseases which are infrequently seen in PAP. Besides the presence of cor pulmonale, fibrotic lung disease from HRCT might be another poor prognostic factor for WLL.

WLL in our study was safe. There were no major postprocedure complications. The most common complication in our series was refractory hypoxemia which might be from the lavage fluid and positive fluid balance. Treatment of refractory hypoxemia included administration of diuretics, suctioning the remaining secretion, optimizing positive end expiratory pressure or using recruitment maneuvers or both, and resuming two-lung ventilation. Although no patients in our study had profound hypoxemia leading to cardiovascular collapse, this complication exists. Indeed, PAP should always be managed in a centre with experience and knowledge. Pneumonia after the procedure was found in $10 \%$; however, all of these patients improved after antibiotics were given without prolonged intubation. All of our patients were discharged from the hospital without using oxygen supplement.

The strengths of our study should be noted. First, our centre is the largest referral centre for WLL in southern Thailand. Second, this is the first study in Thailand which reviewed baseline characteristics of PAP patients and the efficacy of WLL in PAP patients due to the unavailability of inhaled GM-CSF. Last, we collected the data of all patients who underwent WLL for PAP for a long study period with complete follow-up. There are some limitations in our study. First, our study was a retrospective investigation which had some missing data. Second, since we did not test for the anti-GM-CSF antibody, we were not able to report the percentage of autoimmune PAP in our population which was in contrast with previous studies.

\section{Conclusions}

WLL is an effective standard treatment for symptomatic PAP patients. Although the application of the technique varies between expert centres, it is safe and can be used either as the primary treatment or alternative treatment in those settings where inhaled GM-CSF is not available. Our study showed that most of the complications from WLL 
were manageable.

\section{Acknowledgments}

Funding: None.

\section{Footnote}

Reporting Checklist: The authors have completed the STROBE Checklist. Available at http://dx.doi. org/10.21037/jtd-20-3308

Data Sharing Statement: Available at http://dx.doi. org/10.21037/jtd-20-3308

Peer Review File: http://dx.doi.org/10.21037/jtd-20-3308

Conflicts of Interest: Both authors have completed the ICMJE uniform disclosure form (available at http://dx.doi. org/10.21037/jtd-20-3308). The authors have no conflicts of interest to declare.

Ethical Statement: The authors are accountable for all aspects of the work in ensuring that questions related to the accuracy or integrity of any part of the work are appropriately investigated and resolved. The study was conducted in accordance with the Declaration of Helsinki (as revised in 2013). The study was approved by the Office of Human Research Ethics Committee, Faculty of Medicine, Prince of Songkhla University (REC 61-194-14-1) and individual consent for this retrospective analysis was waived.

Open Access Statement: This is an Open Access article distributed in accordance with the Creative Commons Attribution-NonCommercial-NoDerivs 4.0 International License (CC BY-NC-ND 4.0), which permits the noncommercial replication and distribution of the article with the strict proviso that no changes or edits are made and the original work is properly cited (including links to both the formal publication through the relevant DOI and the license). See: https://creativecommons.org/ licenses/by-nc-nd/4.0/.

\section{References}

1. Rosen SH, Castleman B, Liebow AA. Pulmonary alveolar proteinosis. N Engl J Med 1958;258:1123-42.

2. Seymour JF, Presneill JJ. Pulmonary alveolar proteinosis:
Progress in the first 44 years. Am J Respir Crit Care Med 2002;166:215-35.

3. Trapnell BC, Nakata K, Bonella F, et al. Pulmonary alveolar proteinosis. Nat Rev Dis Primers 2019;5:16.

4. Trapnell BC, Whitsett JA, Nakata K. Pulmonary alveolar proteinosis. N Engl J Med 2003;349:2527-39.

5. Borie R, Danel C, Debray MP, et al. Pulmonary alveolar proteinosis. Eur Respir Rev 2011;20:98-107.

6. Lawi D, Dubruc E, Gonzalez M, et al. Secondary pulmonary alveolar proteinosis treated by lung transplant: A case report. Respir Med Case Rep 2020;30:101108.

7. Amital A, Dux S, Shitrit D, et al. Therapeutic effectiveness of rituximab in a patient with unresponsive autoimmune pulmonary alveolar proteinosis. Thorax 2010;65:1025-6.

8. Kavuru MS, Bonfield TL, Thomassen MJ. Plasmapheresis, GM-CSF, and alveolar proteinosis. Am J Respir Crit Care Med 2003;167:1036.

9. Inoue Y, Trapnell BC, Tazawa R, et al. Characteristics of a large cohort of patients with autoimmune pulmonary alveolar proteinosis in Japan. Am J Respir Crit Care Med 2008;177:752-62.

10. Campo I, Mariani F, Rodi G, et al. Assessment and management of pulmonary alveolar proteinosis in a reference center. Orphanet J Rare Dis 2013;8:40.

11. Smith BB, Torres NE, Hyder JA, et al. Whole-lung Lavage and Pulmonary Alveolar Proteinosis: Review of Clinical and Patient-centered Outcomes. J Cardiothorac Vasc Anesth 2019;33:2453-61.

12. Byun MK, Kim DS, Kim YW, et al. Clinical features and outcomes of idiopathic pulmonary alveolar proteinosis in Korean population. J Korean Med Sci 2010;25:393-8.

13. Xu Z, Jing J, Wang H, et al. Pulmonary alveolar proteinosis in China: A systematic review of 241 cases. Respirology 2009;14:761-6.

14. Yang J, Jiang D, Lu JC. Efficacy of the treatment of 10 cases of pulmonary alveolar proteinosis large capacity whole lung lavage. Zhejiang Clin Med J 2014;16:397-8.

15. Gay P, Wallaert B, Nowak S, et al. Efficacy of WholeLung Lavage in Pulmonary Alveolar Proteinosis: A Multicenter International Study of GELF. Respiration 2017;93:198-206.

16. Bonella F, Bauer PC, Griese M, et al. Pulmonary alveolar proteinosis: New insights from a single-centre cohort of 70 patients. Respir Med 2011;105:1908-16.

17. Suzuki T, Trapnell BC. Pulmonary Alveolar Proteinosis 
Syndrome. Clin Chest Med 2016;37:431-40.

18. Briens E, Delaval P, Mairesse MP, et al. Pulmonary alveolar proteinosis. Rev Mal Respir 2002;19:166-82.

19. Zhao YY, Huang H, Liu YZ, et al. Whole Lung Lavage Treatment of Chinese Patients with Autoimmune Pulmonary Alveolar Proteinosis: A Retrospective

Cite this article as: Kaenmuang P, Navasakulpong A. Efficacy of whole lung lavage in pulmonary alveolar proteinosis: a 20year experience at a reference center in Thailand. J Thorac Dis 2021;13(6):3539-3548. doi: 10.21037/jtd-20-3308
Long-term Follow-up Study. Chin Med J (Engl) 2015;128:2714-9.

20. Beccaria M, Luisetti M, Rodi G, et al. Long-term durable benefit after whole lung lavage in pulmonary alveolar proteinosis. Eur Respir J 2004;23:526-31. 